\title{
Super stick, super quick!
}

New Clearfil Universal Bond Quick (UBQ)

from Kuraray Noritake works almost instantly, delivering optimum results without the need to wait. Clearfil UBQ comprises new rapid bond technology, allowing the dental care professional to apply and proceed to the drying stage immediately - no more waiting!

Fine words regarding this new bond, but you could be forgiven for wondering exactly what is beneath the green cap. In this article, we will consider several aspects, providing, among other things, an answer to the following question:

'Why is there no need to wait after the application of Clearfil Universal Bond Quick?'

When a bond is applied to a cavity, it needs to be able to function both on and within the surface of the tooth. Prior to anything else, the bond must penetrate the tooth. During this penetration stage, several ingredients enter the superficial dental tissue. Dentine is the most difficult component in the context of bonding, due to its heterogeneous composition. As dentine contains a high percentage of water (20-25\%), it is logical to use a hydrophilic component to facilitate penetration. A frequently used hydrophilic component is hydroxyethylmethacrylate (HEMA), which can be easily polymerised into PolyHEMA.

In Clearfil Universal Bond Quick, we use a newly developed cocktail of hydrophilic monomers or hydrophilic amide monomers, to be more precise. Kuraray Noritake is renowned for superior bonding techniques and UBQ is no exception. The R\&D

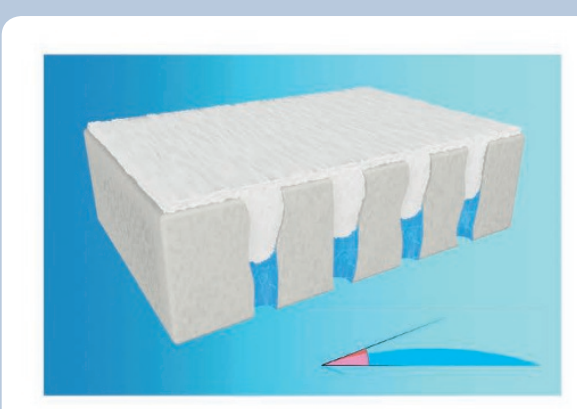

Prepared dentine including smear layer

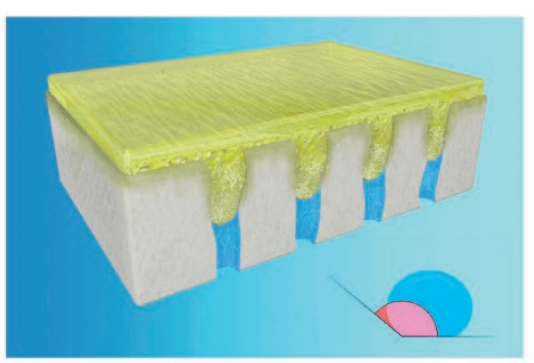

Adhesion to dentine with Clearfil Universal Bond Quick department in Japan put more than 6 years of effort into the development and optimisation of these hydrophilic amide monomers and the results are more than promising.

As the new monomer is much more hydrophilic than HEMA, quick penetration into a hydrophilic surface, such as dentine, is guaranteed. Therefore, first and foremost, this new amide monomer is responsible for rapid penetration. The other components are taken along at the same time and are able to do their work in and on the tooth surface. As a result, waiting times have been almost eliminated.

It is already known PolyHEMA absorbs a lot of moisture; this is a property that the contact lens industry benefits from. A key disadvantage within a bonding system is the occurrence of hydrolysis, which degrades the hybrid layer; therefore, to keep the bonding layer stable, we must ensure, among other things, that hydrolysis does not occur. This is a second property of the cocktail of amide monomers. Due to the creation of a crosslinked, moisture resistant polymer network during light curing, the chance of hydrolysis is greatly reduced.

Curing is optimised by the use of a more advanced photo initiator, which allows the formation of twice as many radicals upon activation of the photo initiator, in comparison to conventional systems. Light curing for ten seconds is therefore enough, with five seconds being suffice when using a higher output lamp (>1,500 MW).

Clearfil Universal Bond Quick also features other impressive properties. Kuraray's original MDP monomer, for example, which ensures immediate adhesion to hydroxyapatite. Calcium-MDP is deposited in the HAp structure, whilst forming nano-layers in a short time. The poor solubility of Ca-MDP stimulates the moisture-resistant properties and, more importantly, long-term adhesion of our bonds to dental tissue - and that includes Clearfil Universal Bond Quick.

Peter Schouten Technical Manager Kuraray Dental Benelux

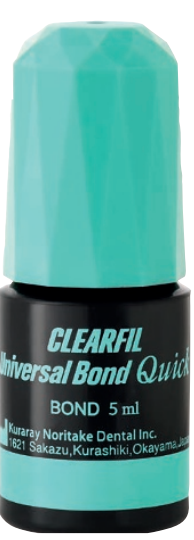

This single-bottle, universal bond works almost instantly, taking just 15 seconds to apply. UBQ contains the MDP monomer, which creates a strong chemical bond to hydroxyapatite, alongside new hydrophilic amide monomers that work together to deliver optimal stability and resistance to moisture for a lasting result. The low film thickness (5-10 $\mu \mathrm{m})$ gives a precise fit, and with 250 applications per $5 \mathrm{ml}$ bottle and a long shelf life, this economic, high strength bond is one that every DCP should have in their drawer. Available through your usual dental dealer or for further information please visit www. js-davis.co.uk, call 01438747344 or email jsdsales@js-davis.co.uk. Clearfil Universal Bond Quick is currently on special offer - Buy 2 get 1 free ( $5 \mathrm{ml}$ bottles). Valid until end June 2017, subject to availability.

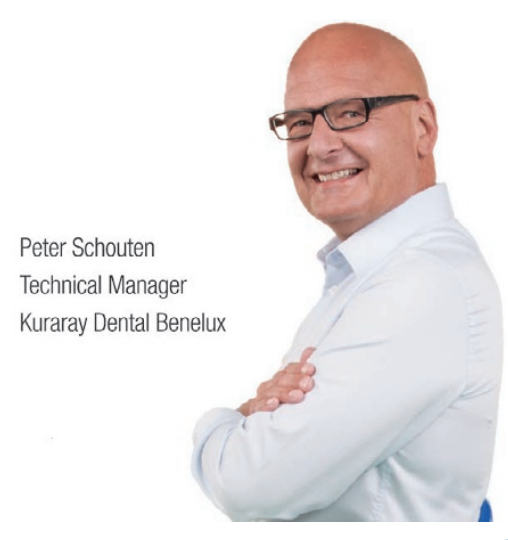

